\title{
Four-Dimensional Ultrasonography of the Fetal Heart Using Color Doppler Spatiotemporal Image Correlation
}

\author{
Luís F. Gonçalves, $M D$, Roberto Romero, MD, Jimmy Espinoza, $M D$, \\ Wesley Lee, MD, Marjorie Treadwell, MD, Kavitha Chintala, MD, \\ Helmut Brandl, Tinnakorn Chaiworapongsa, MD
}

\begin{abstract}
Abbreviations
RA, right atrium; STIC, spatiotemporal image correlation; TR, tricuspid regurgitation; VSD, ventricular septal defect
\end{abstract}

Received December 15, 2003, from the Perinatology Research Branch, National Institute of Child Health and Human Development, National Institutes of Health, Department of Health and Human Services, Bethesda, Maryland and Detroit, Michigan USA (L.F.G., R.R., J.E., T.C.); Department of Obstetrics and Gynecology, Wayne State University/Hutzel Hospital, Detroit, Michigan, USA (L.F.G., M.T., T.C.); Division of Fetal Imaging, William Beaumont Hospital, Royal Oak, Michigan, USA (W.L.); Division of Cardiology, Children's Hospital of Michigan, Wayne State University, Detroit, Michigan USA (K.C.); and General Electric Medical Systems, Kretztechnik, Zipf, Austria (H.B.). Manuscript accepted for publication January 2, 2004.

We thank Jayasree Niyogi for assistance with the literature search, Patrick Schoff for assistance with preparation of the illustrations, Julie Powers for revision and final formatting of the manuscript, and Mike Kammermeier for technical advice on STIC technology.

Address correspondence and reprint requests to Roberto Romero, MD, Perinatology Research Branch, National Institute of Child Health and Human Development, 4707 St Antoine Blvd, Detroit MI 48201 USA.

E-mail:warfiela@mail.nih.gov.

Video online at www.jultrasoundmed.org.
Objective. To describe clinical and research applications of 4-dimensional imaging of the fetal heart using color Doppler spatiotemporal image correlation. Methods. Forty-four volume data sets were acquired by color Doppler spatiotemporal image correlation. Seven subjects were examined: 4 fetuses without abnormalities, 1 fetus with ventriculomegaly and a hypoplastic cerebellum but normal cardiac anatomy, and 2 fetuses with cardiac anomalies detected by fetal echocardiography (1 case of a ventricular septal defect associated with trisomy 21 and 1 case of a double-inlet right ventricle with a 46,XX karyotype). The median gestational age at the time of examination was $213 / 7$ weeks (range, 195/7-34\% weeks). Volume data sets were reviewed offline by multiplanar display and volume-rendering methods. Representative images and online video clips illustrating the diagnostic potential of this technology are presented. Results. Color Doppler spatiotemporal image correlation allowed multiplanar visualization of ventricular septal defects, multiplanar display and volume rendering of tricuspid regurgitation, volume rendering of the outflow tracts by color and power Doppler ultrasonography (both in a normal case and in a case of a double-inlet right ventricle with a double-outlet right ventricle), and visualization of venous streams at the level of the foramen ovale. Conclusions. Color Doppler spatiotemporal image correlation has the potential to simplify visualization of the outflow tracts and improve the evaluation of the location and extent of ventricular septal defects. Other applications include 3-dimensional evaluation of regurgitation jets and venous streams at the level of the foramen ovale. Key words: color Doppler ultrasonography; congenital heart disease; fetal echocardiography; 4-dimensional ultrasonography; prenatal diagnosis; spatiotemporal image correlation; 3-dimensional ultrasonography; ultrasonography.

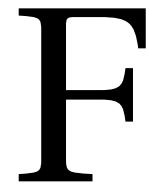

our-dimensional ultrasonography is a technology that adds the temporal dimension (motion) to 3-dimensional imaging. ${ }^{1}$ Spatiotemporal image correlation (STIC) is a recent technological advance that allows 4-dimensional ultrasonographic examination of the fetal heart. ${ }^{2}$ Fetal heart volumes are acquired with a single automated sweep of the transducer. The spatial and temporal information are subsequently combined to display dynamic images that can be extracted from volume data sets. 
Previous research in 4-dimensional ultrasonography using STIC has focused on (1) the study of volume acquisition by an operator with limited skills in the interpretation of cardiac images and post hoc analysis of the volume data sets by a specialist in fetal echocardiography ${ }^{3}$ and (2) development ${ }^{4}$ and validation ${ }^{5}$ of a technique to systematically examine the outflow tracts once a volume of the 4 -chamber view is obtained.

Figure 1. Multiplanar display of a normal fetal heart at 34 weeks. The volume was acquired with an automatic transverse sweep through the fetal chest. Three orthogonal planes are displayed in panels $\mathbf{A}-\mathbf{C}$ (transverse, sagittal, and coronal, respectively). The reference dot, representing the intersection of the 3 orthogonal planes, is positioned at the crux of the heart. 1.A, Diastole. Ventricular filling is seen in panels $\mathbf{A}$ and $\mathbf{C}$ (red). 1.B. Systole. Blood flow is visualized in the ascending aorta and pulmonary artery in panels $\mathbf{B}$ and $\mathbf{C}$ (blue). Note blood flow through the foramen ovale in panel $\mathbf{A}$ (red). Ao indicates ascending aorta; DAo, descending aorta; FO, foramen ovale; IVS, interventricular septum; LA, left atrium; LV, left ventricle; $M B$, moderator band; PA, pulmonary artery; PV, pulmonary veins; RA, right atrium; and $\mathrm{RV}$, right ventricle.

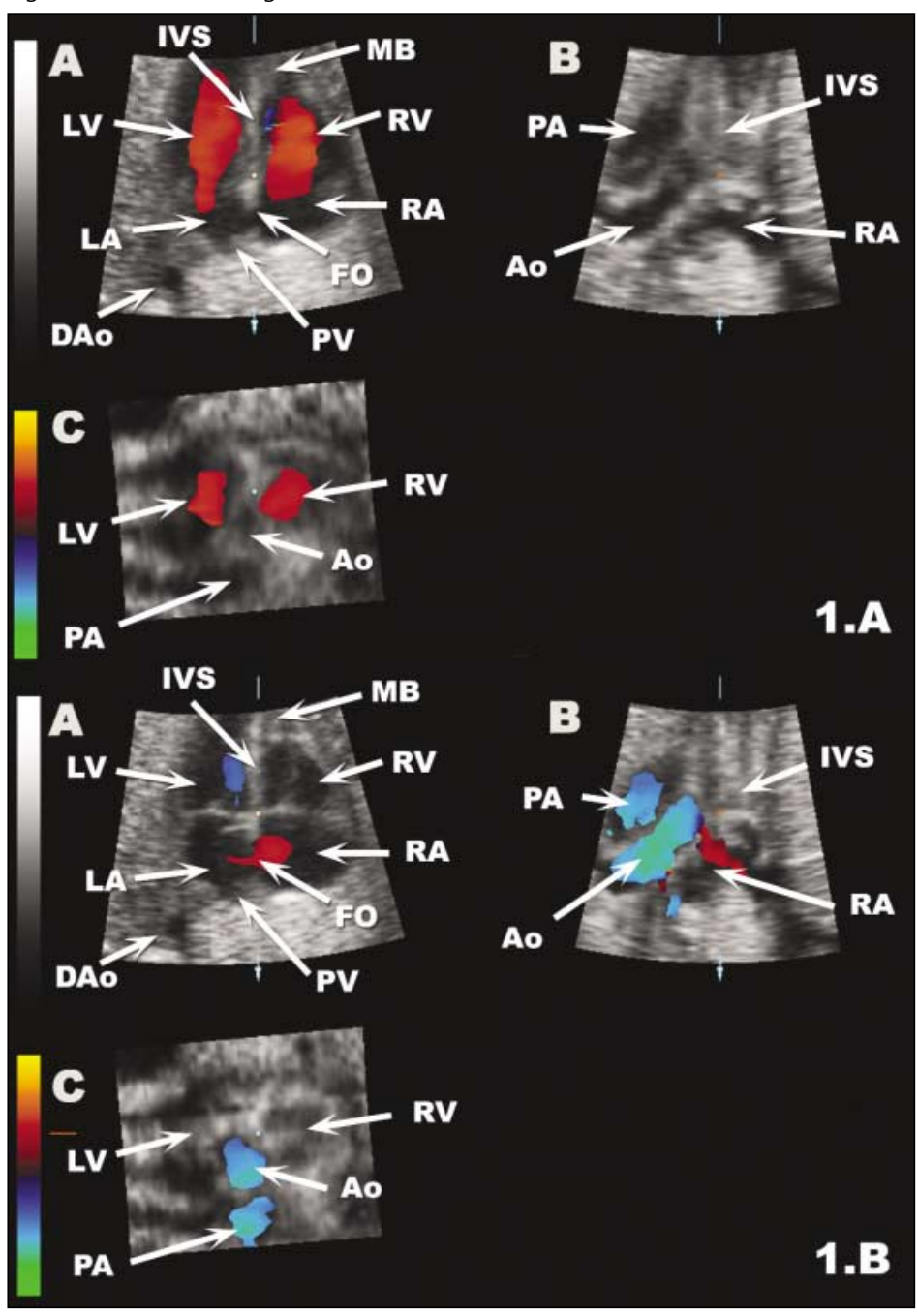

Until recently, only gray scale volumes could be acquired with STIC. A new technological advance adds the possibility of incorporating color and power Doppler information into the volume data set. The objective of this article is to describe clinical and research applications of 4-dimensional imaging of the fetal heart using color Doppler STIC.

\section{Materials and Methods}

Forty-four 4-dimensional volumes of the fetal heart were obtained by STIC with color or power Doppler ultrasonography (Voluson 730 Expert; General Electric Medical Systems, Kretztechnik, Zipf, Austria). Seven subjects were studied: 4 fetuses without abnormalities, 1 fetus with ventriculomegaly and a hypoplastic cerebellum but normal cardiac anatomy, and 2 fetuses with cardiac anomalies detected by fetal echocardiography (1 case of ventricular septal defect associated with trisomy 21 and 1 case of a double-inlet right ventricle, a double-outlet right ventricle, and a $46, \mathrm{XX}$ karyotype). The median gestational age at the time of examination was $21 \frac{3}{7}$ weeks (range, $195 / 7-34 \% / 7$ weeks).

All volumes were acquired with a motorized curved array transducer (2-5 or $4-8 \mathrm{MHz}$ ), with automatic transverse and longitudinal sweeps through the fetal chest and abdomen. The region of interest included the ventricular and atrial chambers, great vessels, and venous return to the fetal heart. Acquisition lasted between 7.5 and 12.5 seconds and, whenever possible, was performed in the absence of fetal movement. Patients were also asked to momentarily suspend breathing.

Images generated from the volume data sets were visualized by multiplanar display and volume-rendering methods. Briefly, the multiplanar display allows dynamic images of the fetal heart to be simultaneously visualized in 3 orthogonal planes (axial, coronal, and sagittal). Volume rendering is a technical process by which 3-dimensional structures of a scanned volume are translated into a 2-dimensional image. Volumerendered images containing gray scale and color Doppler information were displayed with the use of 1 of 3 algorithms: gray (gray scale information only), color (color Doppler information only), or glass body (gray scale and color Doppler information combined). Post processing adjustments in image quality were also performed as neces- 
sary and included (1) gamma curve correction to optimize tissue contrast resolution, (2) color threshold and balance to control the amount of color displayed over bright gray echoes, and (3) gray scale threshold and transparency to improve border recognition of surface-rendered volumes.

Representative images and online video clips illustrating the diagnostic potential of color Doppler STIC are presented. Ultrasonographic studies were conducted under protocols approved by the Institutional Review Boards of Wayne State University and the National Institute of Child Health and Human Development. All patients gave written informed consent before participation in the study.

\section{Results}

\section{Multiplanar Display of the 4-Chamber View: Normal Heart}

Figure 1 (Video 1, 25\% speed reduction) shows multiplanar images of a normal fetal heart at 34 weeks of gestation during diastole (Figure 1A) and systole (Figure 1B). Volume acquisition was performed with an automatic transverse sweep through the fetal chest. The reference dot, which represents the intersection point of the 3 orthogonal planes, was positioned at the crux of the heart. Panel A shows the conventional 4-chamber view with color Doppler imaging; panel B shows a sagittal section through interventricular and interatrial septa; and panel $\mathrm{C}$ shows a coronal section at the level of the atrioventricular valves. Biventricular filling during diastole (red) can be observed in the transverse (Figure 1A, panel A) and coronal (Figure 1A, panel C) planes. Blood flow through the ascending aorta and main pulmonary artery during systole (blue) is visualized in the sagittal (Figure 1B, panel B) and coronal (Figure 1B, panel C) planes. Blood flow through the foramen ovale during systole (red) is observed in the transverse plane (Figure 1B, panel A).

\section{Multiplanar Display of the 4-Chamber View: Ventricular Septal Defect}

A multiplanar display of a ventricular septal defect (VSD) in a fetus with trisomy 21 at 21 weeks of gestation is shown in Figure 2 (Video 2, $25 \%$ speed reduction). The reference dot is positioned at the level of the VSD. Bidirectional blood flow through the VSD can be seen in the 3 orthogonal planes during diastole (Figure 2A) and systole (Figure 2B). Panel A shows the VSD in a transverse 5-chamber view. This is the plane of a section commonly used to show a perimembranous VSD with conventional 2-dimensional ultrasonography. Panel B shows the aspect of the VSD in the sagittal plane, and on panel C, the VSD is shown in a coronal section at the level of the atrioventricular valves.

Figure 2. Multiplanar view of the fetal heart at 21 weeks in a fetus with trisomy 21. Bidirectional flow is shown through the VSD. The original image was rotated over the z-axis to show the transverse section of the heart in the apical 5-chamber view. The color scale is inverted: red represents blood flow toward the transducer, and blue represents blood flow away from the transducer. 2.A, Diastole. A right-to-left shunt through the VSD is shown in red in the transverse (panel $\mathbf{A}$ ), sagittal (panel B), and coronal (panel C) planes of a section. 2.B, Systole. A left-toright shunt through the VSD is shown in blue. Abbreviations are as in Figure 1.

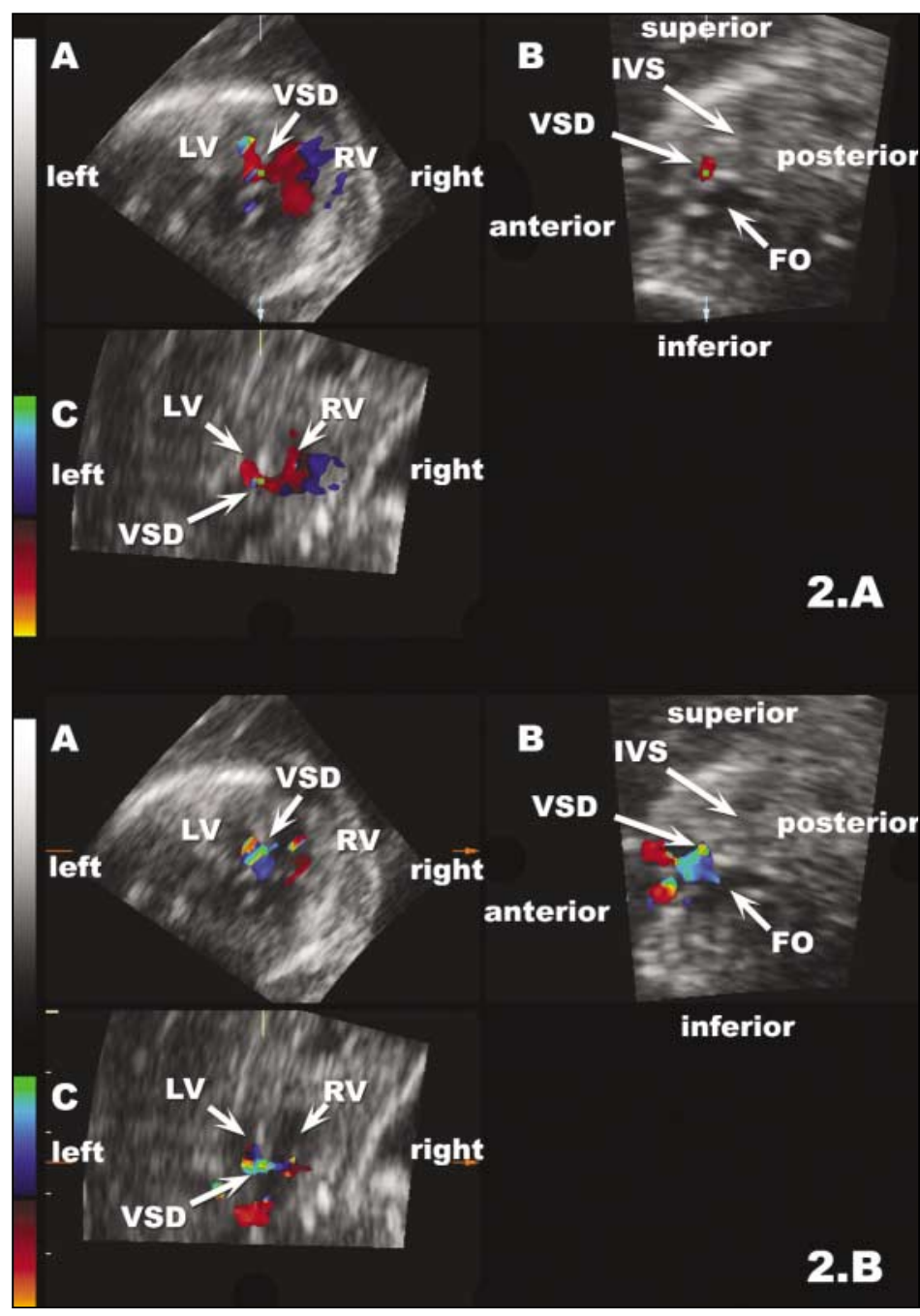




\section{Multiplanar Display and Volume-Rendered Image of Tricuspid Regurgitation}

Figure 3 (video 3, 25\% speed reduction) shows a multiplanar display of a tricuspid regurgitation (TR) jet in a fetus with trisomy 21 at 21 weeks of gestation (panels A-C). This display method allows simultaneous visualization of the regurgitant jet in the transverse, sagittal, and coronal

Figure 3. Multiplanar view of the fetal heart showing TR at 21 weeks in a fetus with trisomy 21. 3.A, Systole. The multiplanar display of the fetal heart during systole allows simultaneous visualization of the TR jet (red) in the transverse (panel A), sagittal (panel B), and coronal (panel C) planes. Panel D shows a volume-rendered image of the TR. This image was produced by first selecting a region of interest with the render box and then applying the glass body rendering algorithm. The direction of view is indicated by the green line in panels $\mathbf{B}$ and $\mathbf{C}$. 3.B, Diastole. Multiplanar (panels A-C) and volume-rendered (panel $\mathbf{D}$ ) images of the fetal heart during diastole are provided for comparison. As expected, the regurgitation jet is not observed during the diastolic phase of the cardiac cycle. Abbreviations are as in Figure 1.

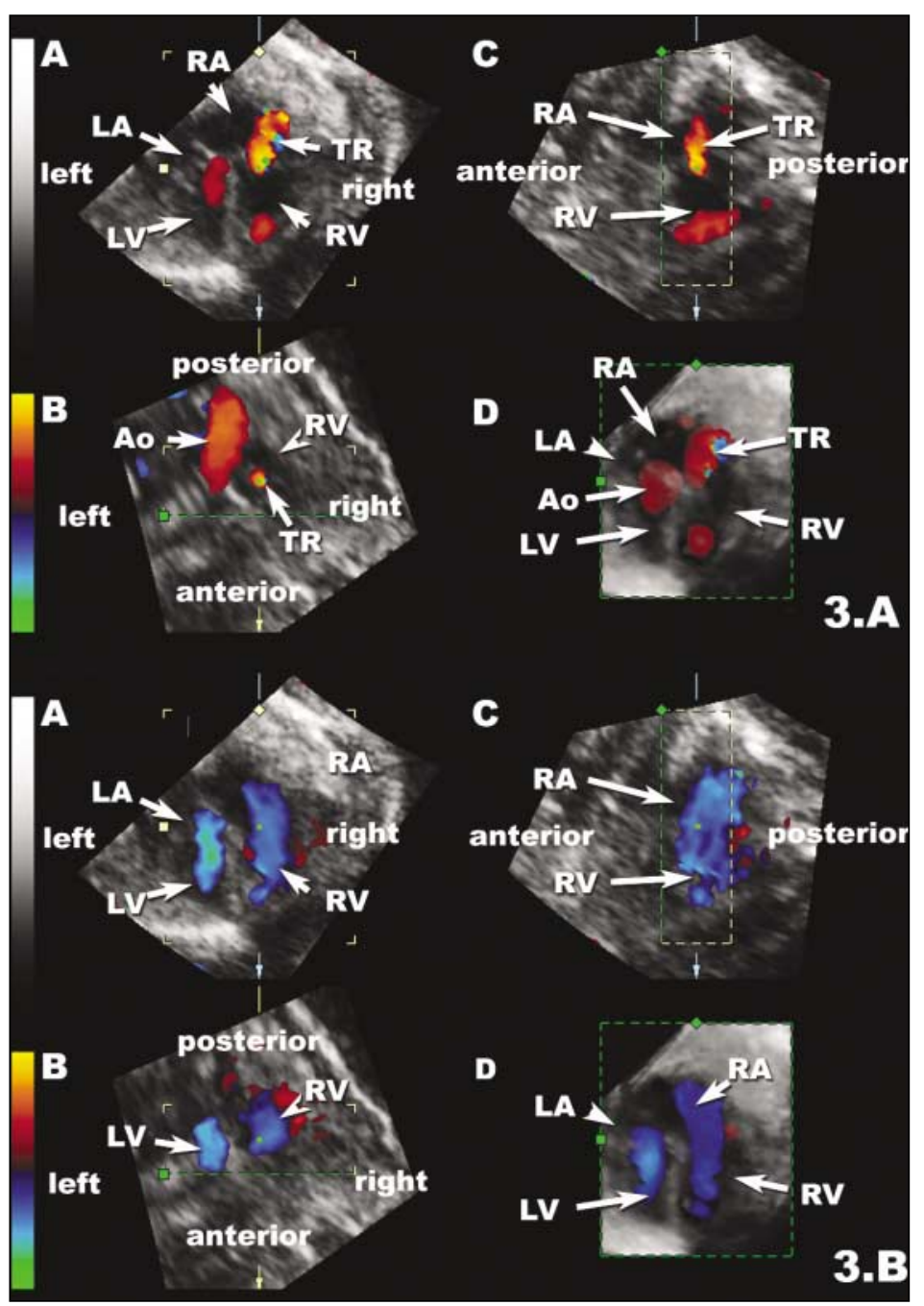

planes. A volume-rendered image of the TR jet is shown on panel $\mathrm{D}$. This image was obtained by adjusting the render box and direction of view over the region of interest shown on panels B and C. Figure 4 shows volume-rendered 3-dimensional images of the TR jet from different views. These images were obtained by rotating the volume $360^{\circ}$ around the y-axis. Snapshots were saved at $0^{\circ}, 90^{\circ}, 180^{\circ}$, and $270^{\circ}$. Panels B and $\mathrm{D}$ show a sagittal view of the TR jet; blood flow is directed toward the posterior wall of the right atrium.

\section{Color and Power Doppler Demonstration of the Normal Outflow Tracts With Volume Rendering}

A novel feature of volume acquisition using color Doppler STIC is 4-dimensional visualization of the normal relationships between the great arteries. Figure 5 (Video 4, 25\% speed reduction) shows a sequence of 6 volume-rendered images of the fetal heart from diastole to systole. The acquisition was performed with an automatic transverse sweep through the fetal chest. The original multiplanar volume, region of interest, and direction of view used to produce the volume-rendered images can be seen in Figure 6 . Figure 7 (Video 5, 25\% speed reduction) shows a similar volume, this time acquired with power instead of color Doppler imaging. With power Doppler imaging, the 3-dimensional shape of the ventricular outlets can be observed during diastole.

\section{Volume Rendering of the Outflow Tracts in a Case of a Double-Inlet Right Ventricle With a Double-Outlet Right Ventricle}

Multiplanar and volume-rendered images in a case of a double-inlet right ventricle with a double-outlet right ventricle at 24 weeks of gestation are presented in Figure 8 (Video 6, 25\% speed reduction). The volume-rendering technique was the same used to analyze the normal heart in Figure 6. Figure 6, panel A, shows a transverse section at the level of the 4-chamber view. A single ventricle is filled during diastole. A sagittal view (panel B), orthogonal to the plane of the section displayed in panel A, shows blood flow through the ascending aorta and pulmonary artery during systole. The volume-rendered image, however, shows only the pulmonary artery. Normal crisscrossing between the 2 outflow tracts is not observed because the 2 vessels 
leave the ventricle parallel to each other, with the pulmonary artery anterior to the aorta (Figure 8B, panel B). Figure 9 shows volume-rendered images of the normal fetal heart during systole (transverse and sagittal, panels A and B, respectively) and comparative images in the case of double-inlet right ventricle with double-outlet right ventricle (transverse and sagittal, panels $\mathrm{C}$ and $\mathrm{D}$, respectively).

\section{Venous Streaming Through the Foramen Ovale}

Volume acquisition with color Doppler STIC can also be used to examine the venous return to the heart. A volume-rendered image (Figure 10 , panel D) and video clip (Video 6, 25\% speed reduction) showing the venous streams originating from the ductus venosus toward the left atrium and from the inferior vena cava toward the right atrium were obtained by limiting the region of interest to the atrial chambers and venous return. The direction of view is from the back of the atrial chambers toward the crux of the heart. In the image presented, the position of the reference dot suggests that preferential streaming from the ductus venosus to the left atrium occurs in the anterior portion of the foramen ovale, closer to the crux of the heart.

\section{Discussion}

Four-dimensional color Doppler ultrasonography of the fetal heart has been described previously. ${ }^{6,7}$ Image acquisition required the simultaneous use of 2 ultrasonography machines and posterior offline analysis in separate computer workstations. Gray scale and color Doppler information were acquired with a $7.5-\mathrm{MHz}$ transducer with 3-dimensional spatial movement tracking, whereas cardiac gating was achieved by simultaneous interrogation of Doppler velocity waveforms from the umbilical artery with another ultrasonographic device. The data sets were reconstructed by a 4-dimensional echo scan machine (TomTec Imaging Systems $\mathrm{GmbH}$, Munich, Germany) and a 3-dimensional imaging workstation developed by the Medical Graphics and Imaging Group, University College London (London, England). Eight volume data sets were successfully acquired in 15 attempts (53.3\%), and among these, $6(75 \%)$ provided useful information. One

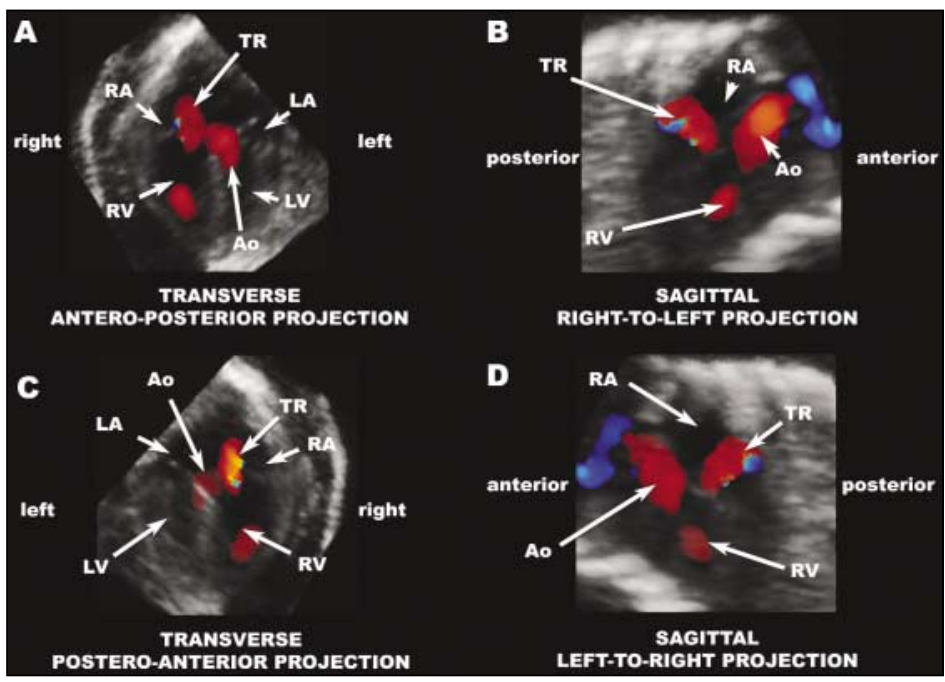

Figure 4. Volume-rendered images of the TR jet in a fetus with trisomy 21 at 21 weeks of gestation. The original volume was the same used to produce the images shown in Figure 3. The volume was rotated $360^{\circ}$ around the $y$-axis, and 4 representative images at $0^{\circ}, 90^{\circ}, 180^{\circ}$, and $270^{\circ}$ are shown. Panel A, Anteroposterior view of the fetal heart. Panel B, Sagittal view of the right ventricle and atrium as seen from the right side of the heart. Note the direction of the TR jet toward the posterior wall of the right atrium. Also observe the ascending aorta projecting in front of the right atrium. Panel $\mathbf{C}$, Posteroanterior view of the fetal heart. Panel D, Sagittal view of the right ventricle and atrium as seen from the left side of the fetal heart. Abbreviations are as in Figure 1.

of the fetuses had a double-outlet right ventricle: the 4-dimensional images depicted both arterial trunks arising from the right ventricle as well as dynamic flow across the interventricular communication. Although the study showed the feasibility of using color velocity Doppler ultrasonography to provide 4-dimensional imaging of fetal cardiac flow, acquisition was hampered by interference generated by the simultaneous use of 2 non-purpose-built transducers and scanners. Other problems included extensive acoustic shadowing, color Doppler angle dependence, and poor border definition of the rendered volumes.

In this article, we describe the diagnostic potential of a new commercially available technique: 4-dimensional ultrasonography with color Doppler STIC. Specifically, we show the feasibility of (1) multiplanar visualization of VSDs, (2) multiplanar display and volume rendering of TR, (3) volume rendering of the outflow tracts by color and power Doppler ultrasonography, and (4) streaming of venous blood flow through the foramen ovale. 


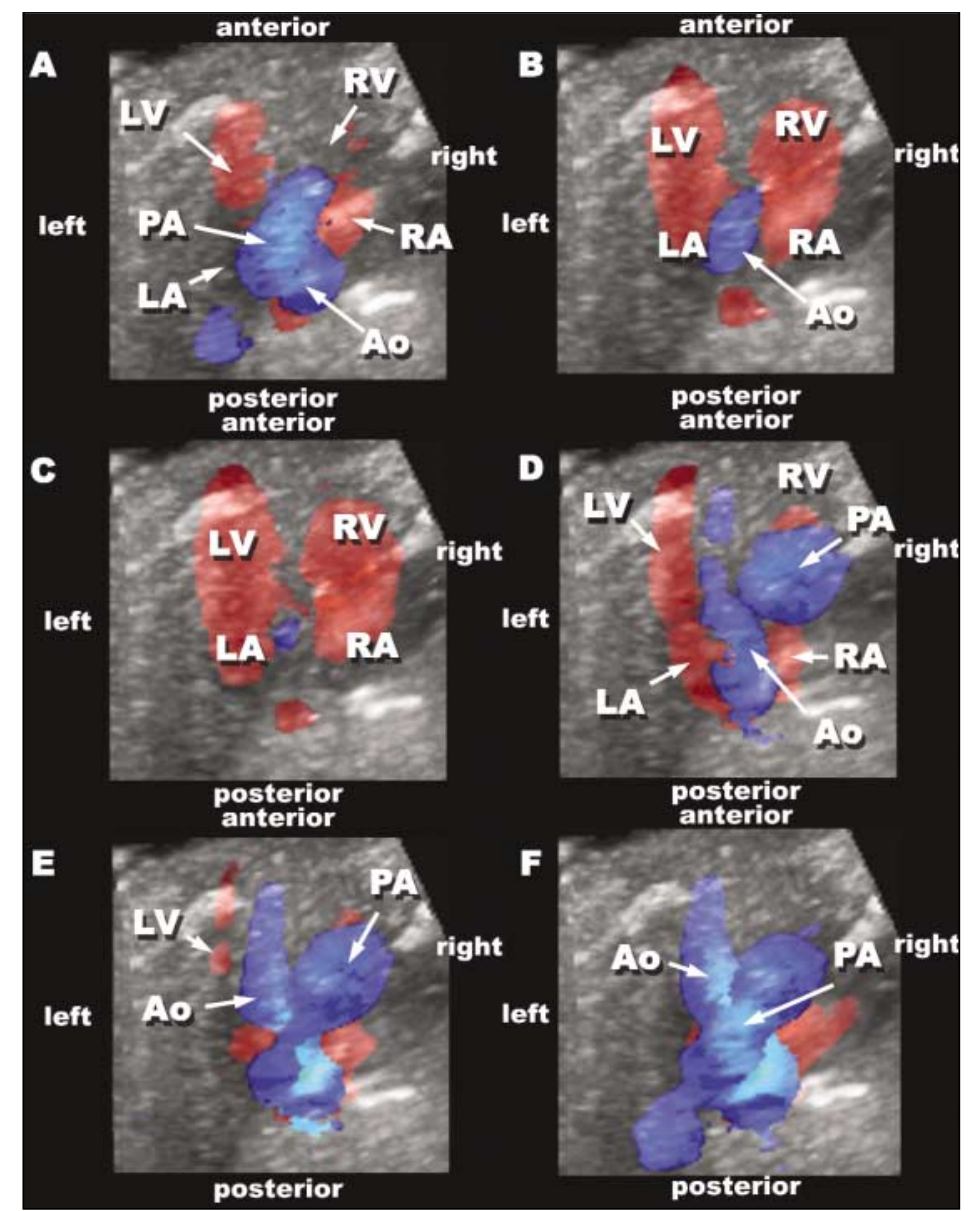

Figure 5. Volume-rendered images of the crisscrossing of the outflow tracts with the use of the glass body algorithm. Panels A-C, Fetal heart during early, mid, and end diastole, respectively. Panels D-F, Fetal heart during early, mid, and end systole. This image allows simultaneous visualization of both outflow tracts from a single volume acquisition with minimal volume manipulation or post processing. Abbreviations are as in Figure 1.

Figure 6. Multiplanar image of the fetal heart during systole. This image illustrates the size and position of the render box as well as the direction of view used to obtain the volume-rendered image of the ventricular outflow tracts. Panel A, Transverse section. Panel B, Sagittal section. Panel C, Coronal section. Panel D, Volume-rendered image of the outflow tracts. Abbreviations are as in Figure 1.

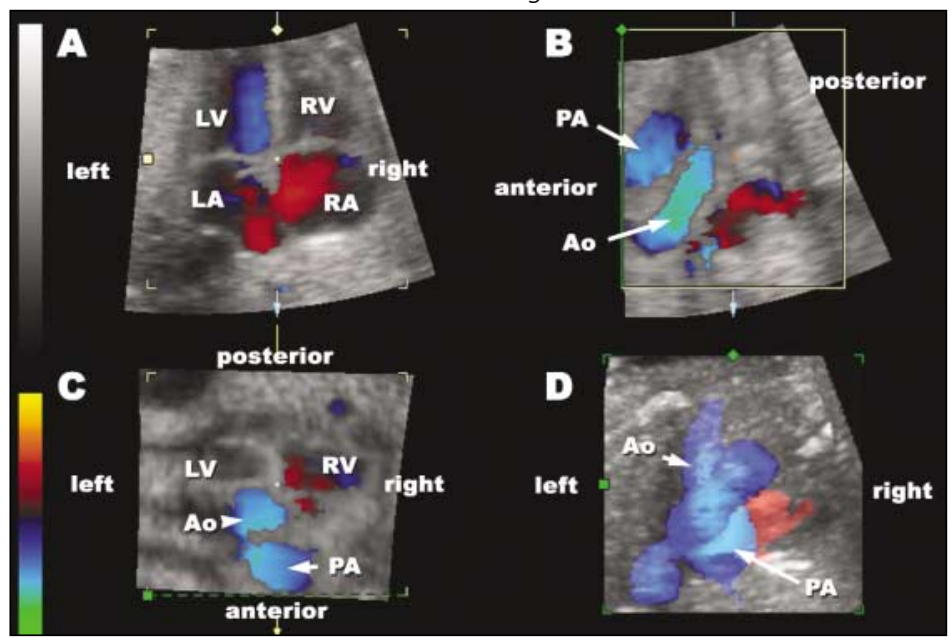

\section{Multiplanar Visualization of VSDs}

Ventricular septal defects are usually diagnosed with the use of transverse planes of the 4- or 5-chamber views. Small VSDs are difficult to diagnose, ${ }^{8-10}$ and the use of color Doppler ultrasonography has been proposed for improving detection rates. ${ }^{11-15}$ Multiplanar analysis of volume data sets acquired with color Doppler STIC (Figure 3) may help in the diagnosis and evaluation of VSDs for the following reasons: (1) once a good-quality volume of the fetal heart is acquired, the difficulties imposed by fetal movement during real-time scanning are eliminated by working directly with the volume data set; (2) heart structures can be imaged with the use of any desired plane of a section; (3) abnormalities that escaped detection during the conventional 2-dimensional scan may be picked up at the time of volume review; (4) images can be reviewed in slow motion or frame by frame if necessary; and (5) simultaneous visualization of the VSD in 3 orthogonal planes may provide a better representation of spatial location and extent.

\section{Three-Dimensional Multiplanar and Volume- Rendered Visualization of TR}

Tricuspid regurgitation is usually suspected during examination of the fetal heart with color Doppler ultrasonography. ${ }^{12,16}$ The severity of TR can be assessed from 2-dimensional and color Doppler flow images obtained in the apical 4chamber view section. The maximal area of the Doppler color flow jet is determined by computerized planimetry, and the area of the right atrium is measured on the same frame. Severity is quantified as the ratio of the Doppler regurgitant jet area to the right atrium (RA) area (the TR/RA ratio). Severe TR is defined as a TR/RA ratio of 0.34 or greater, moderate TR as a TR/RA ratio of 0.20 or greater but less than 0.34 , and mild TR as a TR/RA ratio of less than $0.20 .{ }^{17}$ The significance of 3-dimensional reconstruction of TR jets remains to be determined. Three-dimensional evaluation of the volume, shape, and direction of the regurgitant jet may provide additional information for the assessment of the severity of TR over what is currently possible with 2-dimensional ultrasonography. 


\section{Volume Rendering of the Outflow Tracts}

One of the most promising features of color Doppler STIC is its ability to dynamically display both outflow tracts from a single volume data set of the fetal heart, with no need for substantial volume manipulation. We have previously described and validated a technique to analyze volume data sets acquired with STIC and consistently documented both the long axis view of the left ventricular outflow tract and short axis view of the right ventricular outflow tract in the same multiplanar image. ${ }^{4,5}$ Although simple and reproducible, this technique requires a 4 -step manipulation of the volume data set. Volume rendering of the outflow tracts using color or power Doppler after STIC acquisition takes examination of the outflow tracts to a new level: crisscrossing of the aorta and pulmonary arteries can be visualized with minimal manipulation of the volume data set. Once the 4-chamber view is visualized, the only requirement is to adjust the render box and direction of view, as shown in Figure 6, panels B and C. An example of a case of a double-inlet right ventricle with a double-outlet right ventricle is provided for comparison (Figures 8 and 9); only the pulmonary artery is visualized in the transverse view because the aorta is positioned behind it.

Prenatal diagnosis of outflow tract abnormalities is a desirable goal of prenatal diagnostic imaging. For example, prenatal detection of transposition of the great arteries has a direct impact on reducing the perinatal morbidity and mortality for the affected fetuses. ${ }^{18}$ Unfortunately, the detection rates of abnormalities of the outflow tracts are disappointingly low, especially when prenatal ultrasonography is performed in centers without expertise in fetal echocardiography. ${ }^{8-10}$ Recently, Carvalho et $\mathrm{al}^{19}$ reported improvement in prenatal detection rates for congenital cardiac anomalies in a routine prenatal ultrasonographic screening setting after adding the visualization of the outflow tracts to the scanning protocol. The success of their program, however, relied heavily on an infrastructure committed to continuous in-house training of obstetric sonographers and feedback from fetal cardiologists. Because this kind of infrastructure is not widely available, techniques with the potential to simplify cardiac imaging, such as volume rendering of the outflow tracts using color or power Doppler STIC, may help improve the detection rates for cardiac defects.

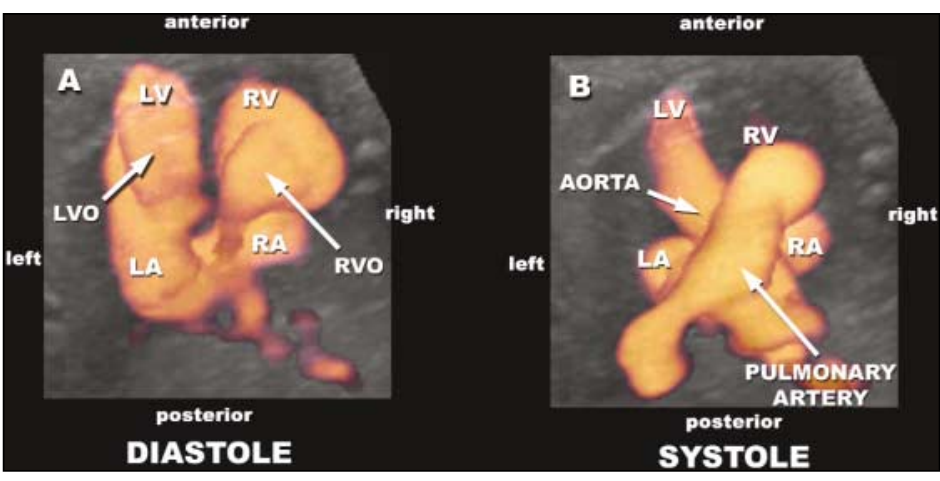

Figure 7. Volume-rendered image of the outflow tracts during diastole (panel $\mathbf{A}$ ) and systole (panel B). The volume was acquired with power Doppler imaging. Note the right and left ventricular outlets (RVO and LVO) clearly visualized at the end of diastole, before opening of the aortic and pulmonary valves. Panel $\mathbf{B}$ shows the normal crisscrossing of the atrioventricular valves during systole. Other abbreviations are as in Figure 1.

Figure 8. Multiplanar image of a fetus with a double-inlet right ventricle and a doubleoutlet right ventricle at 24 weeks of gestation. 8.A, Fetal heart during diastole. 8.B, Fetal heart during systole. Panel $\mathbf{A}$, Transverse section corresponding to the 4-chamber view. Panel B, Sagittal section at the level of the crux of the heart. Panel $\mathbf{C}$, Coronal section through the atrioventricular valves. The single ventricular chamber is best visualized in panels $\mathbf{A}$ and $\mathbf{B}$. A multiplanar view of the great arteries during systole is displayed in 8.B, panel B. Panel D, Volume-rendered image of the fetal heart. The region of interest and direction of view (anteroposterior) are shown in panels $\mathbf{B}$ and $\mathbf{D}$. Abbreviations are as in Figure 1.

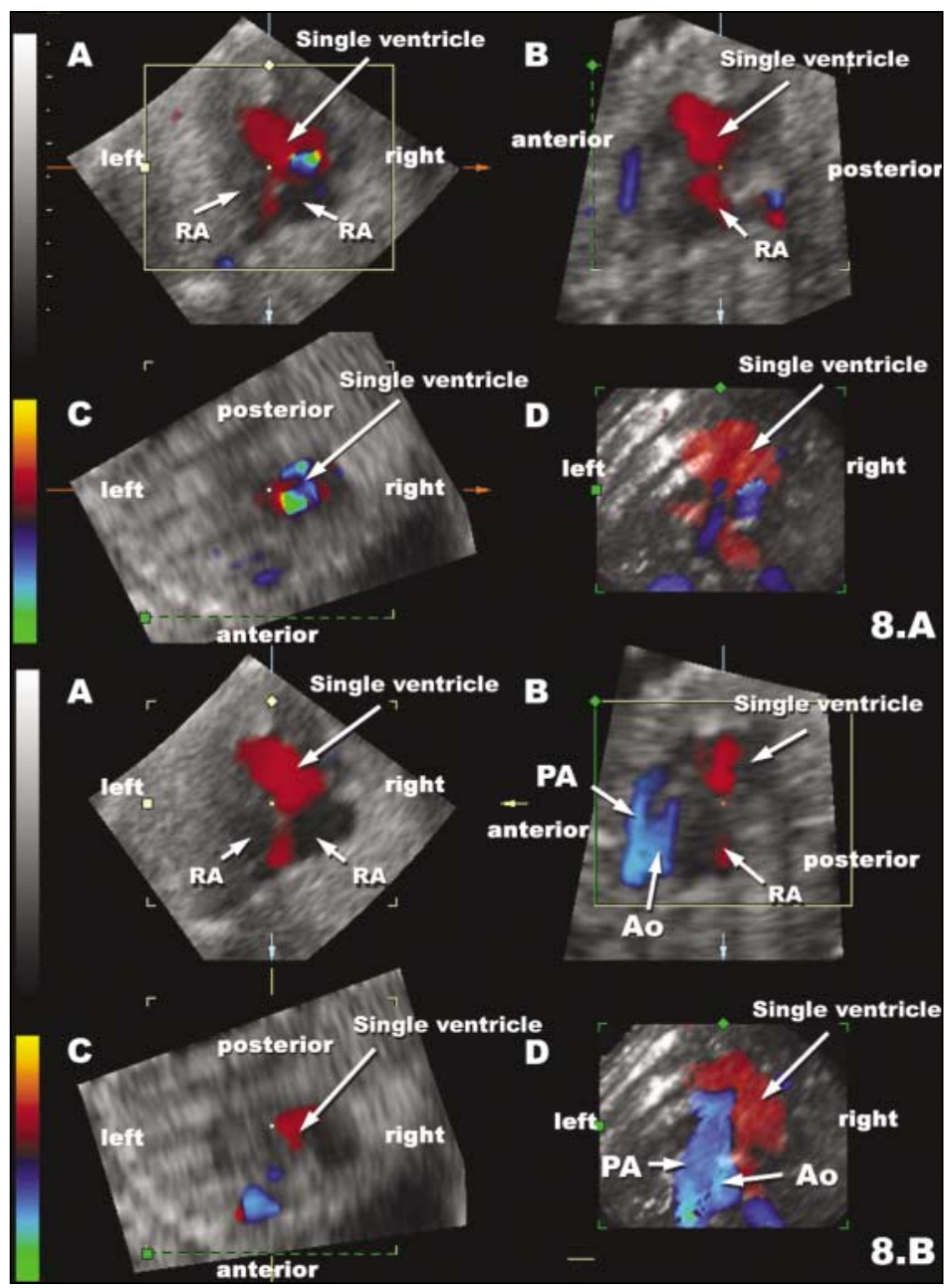




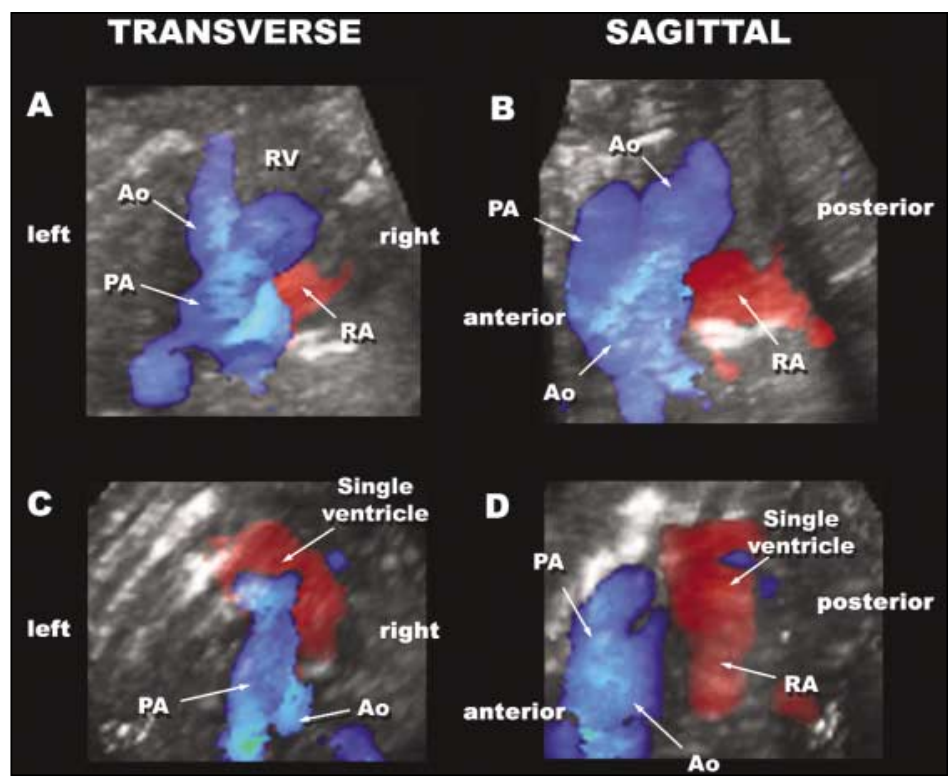

Figure 9. Panels $\mathbf{A}$ and $\mathbf{B}$, Volume-rendered images of the normal outflow tracts in the transverse (panel A) and sagittal (panel B) planes. Panels $\mathbf{C}$ and $\mathbf{D}$, Volume-rendered images of the fetal heart in a case of a double-inlet right ventricle with a double-outlet right ventricle for comparison. Note the crisscrossing of the outflow tracts in the normal heart (panels $\mathbf{A}$ and $\mathbf{B}$ ) and apparently only 1 artery in the case of the double-inlet, double-outlet right ventricle (panel $\mathbf{C}$ ). A small portion of the aorta is actually located behind the pulmonary artery, and the 2 arteries leave the ventricle in parallel (panel $\mathbf{D}$; compare with the normal heart shown on panel $\mathbf{B}$ ). Abbreviations are as in Figure 1.

Figure 10. Volume-rendered image of preferential streaming through the foramen ovale (panel $\mathbf{D}$ ). Streaming is showed by the yellow jet in the center. The red vessel is the inferior vena cava (IVC), and blood flow is directed to the right atrium. High-velocity blood flow from the ductus venosus (blue) is directed through the foramen ovale to the left atrium. Green, indicating turbulent flow, can be observed in 2 points along the path of ductus venosus blood flow: (1) at the point where the high-velocity blood flow from the ductus venosus meets the blood stream originating from the inferior vena cava and (2) at the point of entry into the left atrium. This volume data set was obtained by restricting the region of interest to the atrial chambers and venous return to the right atrium. The render box was positioned at the level of the foramen ovale, and the volume was rotated until all veins of interest (inferior vena cava, ductus venosus, and left hepatic vein) could be visualized in the plane of the section represented on panel B. DV indicates ductus venosus; and LHV, left hepatic vein; other abbreviations are as in Figure 1.

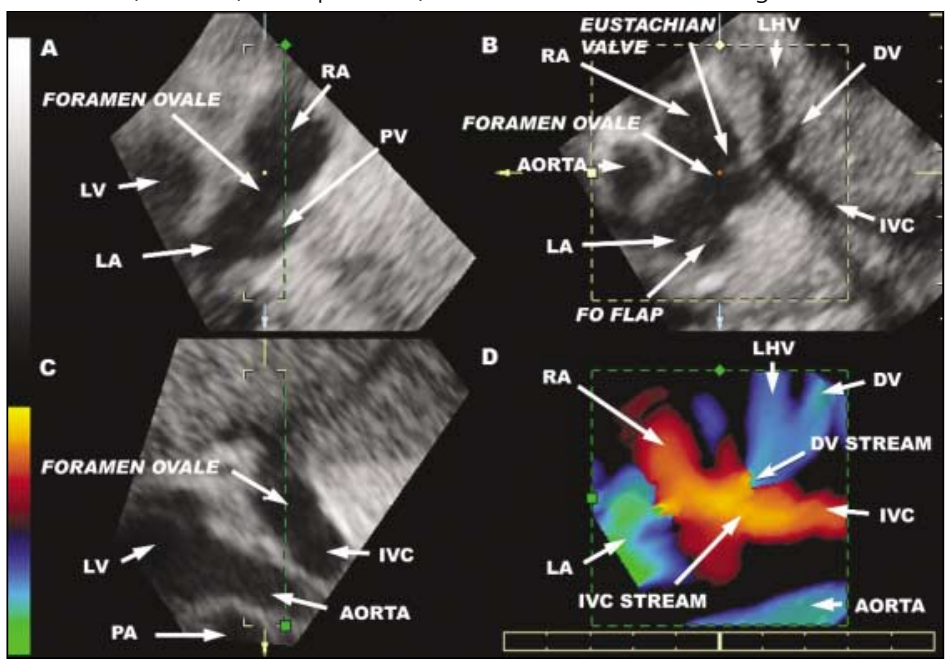

\section{Streaming of Venous Blood Flow Through the Foramen Ovale}

Color Doppler STIC was also used to evaluate the venous return to the heart. Figure 10 shows streaming of blood flow from the ductus venosus to the left atrium through the foramen ovale and from the inferior vena cava to the right atrium. The streams crisscross at the level of the foramen ovale. The position of the reference dot indicates that, at least in the case described in this article, the ductus venosus stream is located in the anterior portion of the foramen ovale, closer to the crux of the heart.

The main intent of this article is to highlight potential new clinical and research applications of color Doppler STIC. More studies are necessary to validate the diagnostic utility of this new technology.

\section{References}

1. Deng J. Terminology of three-dimensional and fourdimensional ultrasound imaging of the fetal heart and other moving body parts. Ultrasound Obstet Gynecol 2003; 22:336-344.

2. DeVore GR, Falkensammer P, Sklansky MS, Platt LD. Spatio-temporal image correlation (STIC): new technology for evaluation of the fetal heart. Ultrasound Obstet Gynecol 2003; 23:380-387.

3. Viñals F, Poblete $P$, Giuliano A. Spatio-temporal image correlation (STIC): a new tool for the prenatal screening of congenital heart defects. Ultrasound Obstet Gynecol 2003; 22:388-394.

4. Gonçalves LF, Lee W, Chaiworapongsa T, et al. Fourdimensional ultrasonography of the fetal heart with spatiotemporal image correlation. Am J Obstet Gynecol 2003; 189:1792-1802.

5. Gonçalves LF, Lee W, Espinoza J, et al. Four-dimensional fetal echocardiography with spatio-temporal image correlation (STIC): a systematic study of standard cardiac views assessed by different observers [abstract]. Ultrasound Obstet Gynecol 2003; 22(suppl 1):50.

6. Deng J, Yates R, Sullivan ID, et al. Dynamic threedimensional color Doppler ultrasound of human fetal intracardiac flow. Ultrasound Obstet Gynecol 2002; 20:131-136.

7. Deng J, Richards R. Dynamic three-dimensional grayscale and Doppler ultrasound of the fetal heart for dynamic diagnosis. Ultrasound Obstet Gynecol 2002; 20:209. 
8. LeFevre $\mathrm{ML}$, Bain RP, Ewigman BG, Frigoletto $\mathrm{FD}$, Crane JP, McNellis D. A randomized trial of prenatal ultrasonographic screening: impact on maternal management and outcome. RADIUS (Routine Antenatal Diagnostic Imaging with Ultrasound) Study Group. Am J Obstet Gynecol 1993; 169: 483-489.

9. Jaeggi ET, Sholler GF, Jones OD, Cooper SG. Comparative analysis of pattern, management and outcome of pre- versus postnatally diagnosed major congenital heart disease: a population-based study. Ultrasound Obstet Gynecol 2001; 17:380-385.

10. Garne E, Stoll C, Clementi M, Euroscan Group. Evaluation of prenatal diagnosis of congenital heart diseases by ultrasound: experience from 20 European registries. Ultrasound Obstet Gynecol 2001; 17:386391.

11. Kurjak A, Breyer B, Jurkovic D, Alfirevic Z, Miljan M. Color flow mapping in obstetrics. J Perinat Med 1987; 15:271-281.

12. DeVore GR, Horenstein J, Siassi B, Platt LD. Fetal echocardiography, VII: Doppler color flow mapping: a new technique for the diagnosis of congenital heart disease. Am J Obstet Gynecol 1987; 156:1054-1064.

13. Gembruch U, Chatterjee MS, Bald R, Redel DA, Hansmann M. Color Doppler flow mapping of fetal heart. J Perinat Med 1991; 19:27-32.

14. Lethor JP, Marcon F, de Moor M, King ME. Physiology of ventricular septal defect shunt flow in the fetus examined by color Doppler M-mode. Circulation 2000; 101:E93.

15. DeVore GR. Trisomy 21: $91 \%$ detection rate using second-trimester ultrasound markers. Ultrasound Obstet Gynecol 2000; 16:133-141.

16. Respondek ML, Kammermeier M, Ludomirsky A, Weil SR, Huhta JC. The prevalence and clinical significance of fetal tricuspid valve regurgitation with normal heart anatomy. Am J Obstet Gynecol 1994; 171:12651270.

17. Hinderliter AL, Willis PW IV, Long WA, et al. Frequency and severity of tricuspid regurgitation determined by Doppler echocardiography in primary pulmonary hypertension. Am J Cardiol 2003; 91:1033-1037, A9.

18. Bonnet D, Coltri A, Butera G, et al. Detection of transposition of the great arteries in fetuses reduces neonatal morbidity and mortality. Circulation 1999; 99:916-918.
19. Carvalho JS, Mavrides E, Shinebourne EA, Campbell $S$, Thilaganathan B. Improving the effectiveness of routine prenatal screening for major congenital heart defects. Heart 2002; 88:387-391. 